\title{
Evaluation of the effects of Uncaria rhynchophylla alkaloid extract on LPS-induced preeclampsia symptoms and inflammation in a pregnant rat model
}

\author{
Liang-Zhi Wu (iD) ${ }^{1,2}$ and Xiao-Min Xiao (iD)
}

${ }^{1}$ Department of Obstetrics and Gynecology, 1st Affiliated Hospital of Jinan University, Guangzhou, Guangdong, China ${ }^{2}$ Department of Obstetrics and Gynecology, Guangdong Second Provincial General Hospital, Guangzhou, Guangdong, China

\begin{abstract}
Excessive pro-inflammatory cytokines result in adverse pregnancy outcomes, including preeclampsia-like phenotypes, and fetal growth restriction. Anti-inflammation might be an effective therapy. The aim of this research was to investigate whether Uncaria rhynchophylla alkaloid extract (URE), a highly safe anti-inflammation constituent of the herb, can inhibit inflammation and improve clinical characteristics of preeclampsia in a lipopolysaccharide (LPS)-induced preeclampsia rat model. The rat model was established by daily administration of LPS (1 $\mu \mathrm{g} / \mathrm{kg}$ body weight per day) from gestational day (GD) 14 to 19 . Different doses of URE (35, 70, and $140 \mathrm{mg} / \mathrm{kg}$ body weight per day) were administered from GD 14 to GD 19. The effects of URE on proteinuria, maternal hypertension, pregnancy outcomes, as well as pro-inflammatory cytokines levels in serum and placenta were measured. High-dose URE (HURE) treatment decreased LPS-induced mean 24-h proteinuria and systolic blood pressure, and increased fetal weight, placental weight, and the number of live pups $(P<0.05)$. Moreover, increased serum and placental levels of interleukin (IL)-6, IL-1 $\beta$, tumor necrosis factor- $\alpha$, and interferon- $\gamma$ in the LPS-treated group were obviously inhibited after HURE administration $(P<0.01)$. URE improved preeclampsia symptoms and mitigated inflammatory responses in the LPSinduced preeclampsia rat model, which suggests that the anti-inflammation effect of URE might be an alternative therapy for preeclampsia.
\end{abstract}

Key words: Preeclampsia; Inflammation; Uncaria rhynchophylla; Alkaloids

\section{Introduction}

Preeclampsia $(\mathrm{PE})$ is a pregnancy-specific multisystem disorder and is characterized by proteinuria, hypertension, unexplained seizure, and severe headache (1). The manifestations of fetal PE include abnormal fetal growth, fetal oxygenation, and reduced amniotic fluid (2). This is a severe obstetric problem, of which the definite etiology is still unclear. PE affects about $5 \%$ of pregnancies and is a leading cause of maternal morbidity and mortality (3). Insulin resistance, increased systemic inflammatory response, and endothelial dysfunction are also present in PE (4). Although the main cause that triggers these dysfunctions in PE remains unknown, accumulating evidence shows that abnormal maternal-fetal inflammatory response and immune system activation play a significant role in the pathogenesis of PE (5). Alterations in maternal peripheral blood show that pregnancy is related to a mild yet sustained systemic inflammatory response (6). Recent research has also reported decreased serum levels of anti-inflammatory cytokines and increased serum levels of pro-inflammatory cytokines in PE women compared to normal pregnancy (7).
Despite PE being considered an exaggerated inflammatory response resulting from a changed immune response, there is no effective and innovative treatment for PE.

Natural medicines have displayed positive effects on blood pressure and proteinuria in lipopolysaccharide (LPS)-induced PE rats (8). Similarly, administration of the extract from Vitis labrusca causes insulin resistance, a decrease in blood pressure, and increased the numbers of live fetuses in L-NAME-induced rats (9). Therefore, the search for herbal medicines with lower toxicity and efficient effects is important for developing novel therapy for PE treatment.

The herb Uncaria rhynchophylla, also known as Gou-teng, is an important traditional Chinese medicine generally used for the treatment of fever, bilious disorders, and dizziness for years in China. The leaves and stems of $U$. rhynchophylla are also used to treat nervous disorders and cerebrovascular diseases associated with epilepsy, hypertension, and preeclampsia (10). Indole alkaloids are the major active components of $U$. rhynchophylla extracts 
that have the anti-epilepsy, anti-hypertensive, and antiinflammatory activities (11-13). U. rhynchophylla exhibits neuroprotective activities via the suppression of COX-2 and the subsequent decrease of inflammation in rats (14). Rhynchophylline, one of the main components of $U$. rhynchophylla, exhibits anti-convulsive and neuroprotection effects (15). In addition, $U$. rhynchophylla and rhynchophylline displayed anti-convulsive activities in kainic acid-induced rats via the suppression of interleukin (IL)-1 $\beta$ and brain-derived neurotrophin factor gene expressions (11). Moreover, U. rhynchophylla displayed inhibitory activities on LPS-induced IL-1 $\beta$ and NO production in macrophages (16). IL-1 $\beta$ and other inflammatory cytokines such as tumor necrosis factor- $\alpha$ (TNF- $\alpha$ ), IL-6, and interferon- $\gamma($ IFN- $\gamma)$ may be associated with the pathogenesis of PE $(17,18)$.

LPS, a commonly known endotoxin, is a toxic component of Gram-negative bacterial cell walls, which has been linked to preeclampsia, fetal growth restriction, and embryonic resorption $(19,20)$. Gram-negative bacterial infections are a cause of preterm labor and fetal loss in humans (21). Establishing maternal infection by injecting pregnant rodents with LPS led to an increase of inflammatory levels in the placenta and gave rise to preeclampsia, fetal loss, and fetal growth restriction (22). Hence, anti-inflammation might be an effective therapy for the prevention and treatment of these complications (23).

Therefore, the purpose of the present research was to investigate whether $U$. rhynchophylla alkaloid extract (URE) could improve PE-like symptoms in a LPS-induced rat model via suppression of pro-inflammatory cytokines. In addition, the alkaloids composition of URE was characterized using HPLC-MS methods. This study aimed to further characterize the possible functional components that are responsible for its anti-hypertensive and antiinflammatory activities.

\section{Material and Methods}

\section{Reagents and materials}

Dried $U$. rhynchophylla was purchased from Guang Dong Feng Chun Pharmaceutical Co., Ltd. (China). Ultrapure LPS (strain Escherichia coli 055: B5), isorhynchophylline, yohimbine, $3 \alpha$-dihydrocadambine, raubasine, hirsuteine, and hirsutine were purchased from SigmaAldrich (USA). Enzyme-linked immunosorbent assay (ELISA) kits specific for IFN- $\gamma$, IL-6, IL-1 $\beta$, and TNF- $\alpha$ were purchased from eBioscience (USA).

\section{Preparation of URE}

Dried U. rhynchophylla (500 g) was extracted four times with $500 \mathrm{~mL}$ of $50 \%$ ethanol. After filtration, the extract was evaporated and condensed under a vacuum condition. Next, the concentrate was dissolved in $50 \mathrm{~mL}$ water and added into the NKA-II macroreticular resin column for adsorption for $16 \mathrm{~h}$. The column was washed with water and $20 \%$ ethanol, in order to get rid of impurities. URE was refined through elute with $95 \%$ ethanol. The eluate was collected and condensed in vacuum, and then further dried for $24 \mathrm{~h}$ at $-20^{\circ} \mathrm{C}$ using a freeze-dryer (24).

\section{HPLC/MS analysis of URE}

The alkaloids present in the URE were identified by high performance liquid chromatography-mass spectrometry (HPLC-MS) using an Agilent 1260 HPLC system equipped with an Agilent 6400 MSD (USA) with the electrospray source operated in the positive ion mode. Samples containing $0.5 \mathrm{mg} / \mathrm{mL}$ of URE in acetonitrile were passed through a $0.22 \mu \mathrm{m}$ filter, and aliquots $(1 \mu \mathrm{L})$ were injected onto Agilent Eclipse Plus C18 column $(4.6 \times 50 \mathrm{~mm}$, $1.8 \mu \mathrm{m}, 600$ bar). Gradient elution was carried out using a mixture of $0.1 \%$ formic acid in water $(A)$ and $100 \%$ acetonitrile (B) supplied at a flow rate of $0.4 \mathrm{~mL} / \mathrm{min}$. Detection was performed at $254 \mathrm{~nm}$. The mobile phase was kept at $95 \%$ A between 0 and $0.5 \mathrm{~min}$, followed by a linear gradient from 95 to $30 \%$ A between 0.5 and 5 min, and subsequently kept at $30 \%$ A between 5 and $10 \mathrm{~min}$.

\section{Animal experimental design}

The animal experimental protocols were approved by the Animal Ethics Committee of the Guangdong Second Provincial General Hospital and all animal studies were carried out according to Guide for the Care and Use of Experimental Animals. The 6-8-week-old female SpragueDawley rats (weighing 180-210 g) were purchased from the Experimental Animal Center of Hunan province and housed under laboratory temperature of $22-24^{\circ} \mathrm{C}$, relative humidity 50-60\%, illumination between 06:00 am and 06:00 pm with free access to basal diets and water. After a week of acclimatization, all female rats were mated with fertile males at a 1:1 ratio, and a positive vaginal smear for sperm defined gestational day (GD) 0 . A total of 40 female rats were randomly divided into five groups (8 rats each group): pregnant control group (PC), LPS-treated control group (MC), LPS plus high-dose URE group (HURE, $140 \mathrm{mg} / \mathrm{kg}$ body weight per day), LPS plus medium-dose URE group (MURE, $70 \mathrm{mg} / \mathrm{kg}$ body weight per day), and LPS plus low-dose URE group (LURE, $35 \mathrm{mg} / \mathrm{kg}$ body weight per day). The experimental PE rats were induced by injection of LPS $(1 \mu \mathrm{g} / \mathrm{kg}$ body weight per day, dissolved in $2 \mathrm{~mL}$ saline) on GD 14 (25). Intragastric administration was carried out once a day for all rats from GD 14 to 19. Rats in the PC group and $\mathrm{MC}$ group were intragastrically administered an equivalent volume of saline.

\section{Measurement of cytokines}

On GD 20, blood samples were collected from the inferior vena cava for cytokine measurement. Afterwards, all rats were euthanized. The placentas and pups were weighed. All samples were kept at $-20^{\circ} \mathrm{C}$ for further analyses. Total lysates were obtained by homogenizing $100 \mathrm{mg}$ of placenta in $1000 \mu \mathrm{L}$ of RIPA lysis buffer. The levels of 
serum and placental cytokines (IL-6, IL-1 $\beta$, TNF- $\alpha$, IFN- $\gamma$ ) were quantified by a commercial ELISA kit (Bio-Rad, USA) according to the manufacturer's instructions. All samples were determined in duplicate. The data analysis was performed using Bio-Plex Manager (Version 5.0, Bio$\mathrm{Rad})$, and data are reported as concentrations $\left(\mathrm{pg} \cdot \mathrm{mL}^{-1}\right.$. $\mathrm{mg}^{-1}$ or $\mathrm{pg} / \mathrm{mL}$ ).

\section{Measurement of systolic blood pressure (SBP) and proteinuria}

On GDs 6, 10, 14, 16, and 18 (between 8 a.m. and 10 a.m.), the SBP was measured with a tail cuff sphygmomanometer (CODA, Kent Scientific, USA). All rats were warmed to $38^{\circ} \mathrm{C}$ for adapting to the experimental conditions before the actual measurements were performed. For each rat, the value of SBP was measured continuously 12 times, and three continuous values varying less than $6 \mathrm{mmHg}$ were averaged to record the SBP. On GDs $7,12,17$, and 19 , each rat was housed in an individual metabolic cage to obtain the urine sample. Urine samples were centrifuged at $5000 \mathrm{~g}$ for $10 \mathrm{~min}$ at $22^{\circ} \mathrm{C}$, and the supernatant was obtained for further analysis of the protein content. The protein level was measured with the pyrogallol red method (26).

\section{RNA isolation and real-time polymerase chain reaction (RT-PCR)}

Total RNA was extracted from placenta tissues using TRIzol reagent (Invitrogen, USA), according to the manufacturer's instructions. cDNA synthesis was carried out by reverse transcription of $1 \mu \mathrm{g}$ of purified RNA using First Strand cDNA Synthesis kit (Takara, China). RT-PCR amplification was performed with the SYBR Green qPCR Master Mix kit (Thermo, USA), following the manufacturer's protocol. The qPCR was performed in duplicate, using GAPDH as the reference gene. The condition of RTPCR amplification reaction was as follows: 40 cycles of $95^{\circ} \mathrm{C}$ for $10 \mathrm{~s}, 60^{\circ} \mathrm{C}$ for $15 \mathrm{~s}$, and $72^{\circ} \mathrm{C}$ for $30 \mathrm{~s}$ with the specific primer sequences (Table 1 ). Data analysis was carried out using the $2^{-\Delta \Delta C T}$ method.

\section{Immunohistochemistry staining analysis of the expression of NF-кB p65}

The collected placental tissues were fixed in $10 \%$ formalin and embedded in paraffin. The antigen retrieval of placental tissue sections (4- $\mu \mathrm{m}$ thickness) was carried out under high pressure after deparaffinization. Tissues were immersed in $0.3 \%$ hydrogen peroxide for $30 \mathrm{~min}$ to block the nonspecific antigens and endogenous peroxidase activity. Then, the tissue sections were incubated for 30 min with $5 \%$ goat serum, and subsequently incubated with rabbit anti-NF-KB p65 antibodies (1:50 dilution) in a humidified chamber overnight at $4^{\circ} \mathrm{C}$. After washing with PBS, the sections were incubated with rabbit antigoat antibody for 20 min and were subsequently incubated with horseradish peroxidase and then counterstained with hematoxylin. The tissues were analyzed under a brightfield microscope. The degree of NF- $\kappa \mathrm{B}$ expression was indicated as the percentage of nuclear NF- $\mathrm{BB}$ p65-positive cells out of the total number of placental cells.

\section{Statistical analysis}

All experimental data are reported as means \pm SE. Data analysis among multiple groups was performed by one-way analysis of variance (ANOVA) followed by the least significant difference post hoc test or Dunnett's test. Data were analyzed by SPSS 19.0 software (SPSS, USA). $P$-values of $<0.05$ were regarded as statistically significant.

\section{Results}

\section{Chemical characteristics of oxindole alkaloids from URE}

Firstly, the amounts of total oxindole alkaloids were quantified in order to standardize the URE. Of the URE total alkaloid content, $66.3 \%$ was of oxindole alkaloids. The chromatograms of alkaloids occurring in URE detected at $254 \mathrm{~nm}$ is displayed in Figure 1. The qualitative identification using standard reference solutions confirmed the presence of isorhynchophylline, yohimbine, $3 \alpha$-dihydrocadambine, raubasine, hirsuteine, and hirsutine. The six constituents were supported by previous research (27). Detailed HPLC-MS data of these constituents are shown in Figure 2.

\section{URE reduced the hypertension in the LPS-induced PE-like rats}

As displayed in Figure 3, SBP in the PC group did not change significantly during the study period. There were

Table 1. Primer sequences for quantitative real-time RNA.

\begin{tabular}{|c|c|c|}
\hline Genes & Forward primer & Reverse primer \\
\hline IL-6 & 5'-TGGTGATAAATCCCGATGAAG-3' & 5'-GGCACTGAAACTCCTGGTCT-3' \\
\hline IL-1 $\beta$ & 5'-GCCGATGGTCCCAATTACAT-3' & 5'-ACAAGACCTGCCGGAAGCT-3' \\
\hline TNF- $\alpha$ & 5'-СССТСАСАСТСАGATCATCTTCT-3' & 5'-GCTACGACGTGGGCTACAG-3' \\
\hline $\mathrm{IFN}-\gamma$ & 5'-TGAGCCAGATTGTTTCGATG-3' & 5'-TCCTTTTGAAACTCGGAGGA-3' \\
\hline GAPDH & 5'-CAACTTTGGCATTGTGGAAGG-3' & $5^{\prime}$-ACACATTGGGGGTAGGAACAC- $3^{\prime}$ \\
\hline
\end{tabular}




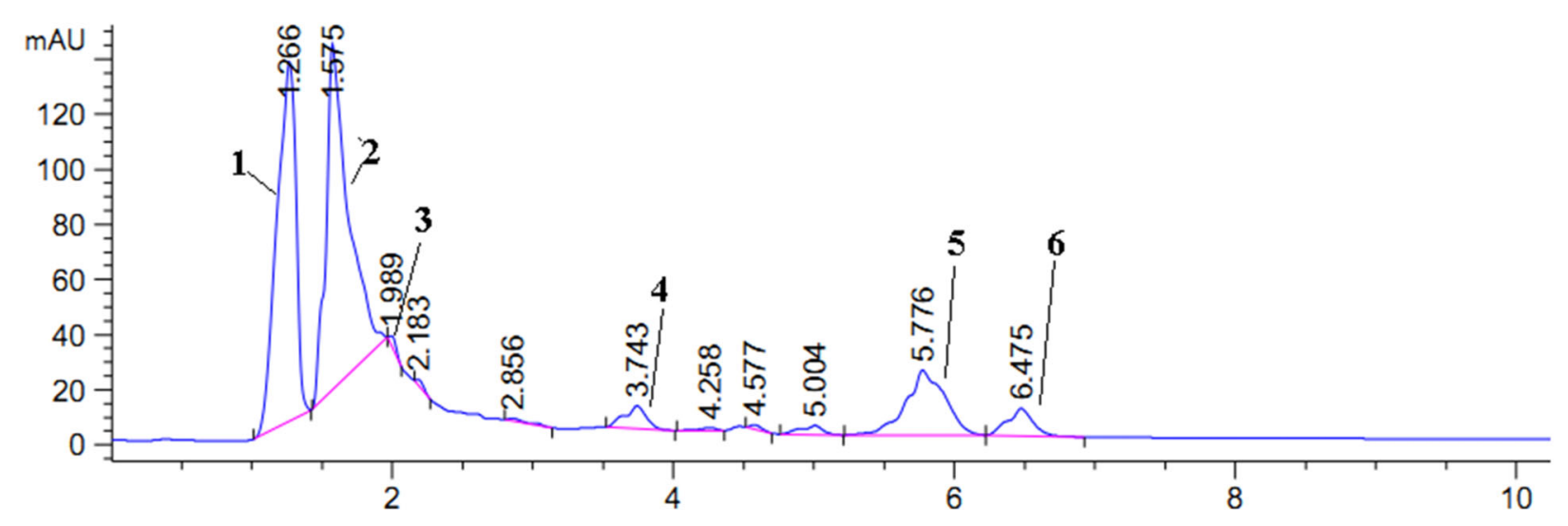

Figure 1. Chromatograms of alkaloids occurring in Uncaria rhynchophylla extract. 1: isorhynchophylline; 2: yohimbine; 3: 3 $\alpha$-dihydrocadambine; 4: raubasine; 5: hirsuteine; 6: hirsutine.
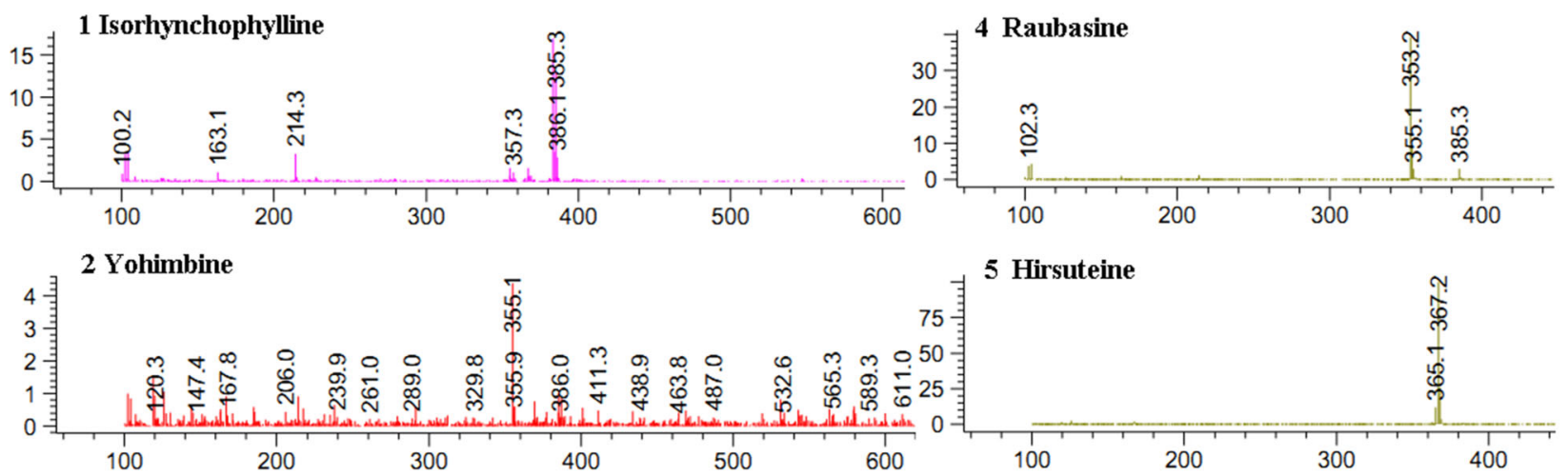

5 Hirsuteine
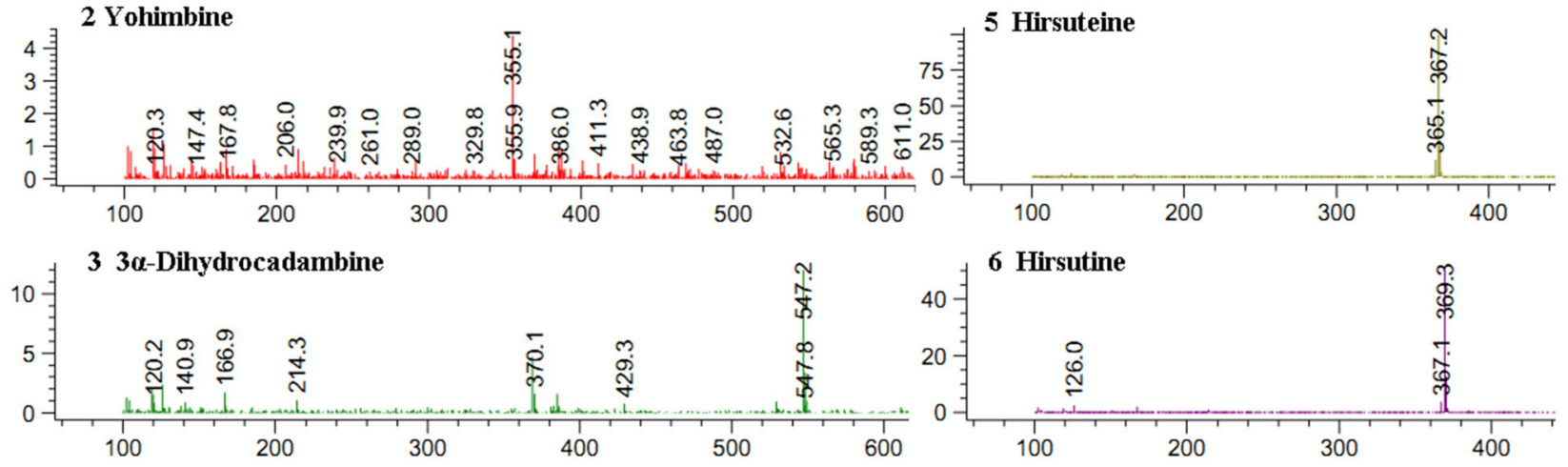

Figure 2. Positive fragments of alkaloids present in Uncaria rhynchophylla extract and characterization of six representative components assessed by HPLC-MS.

no differences in SBP profiles among the five groups before GD 14, but it was significantly increased in LPSinduced rats on GD 16 and 18 compared with the PC group $(P<0.01)$. However, the values of SBP were significantly lower in the treated rats than those in the untreated rats (HURE vs MC, $100.33 \pm 5.85 \mathrm{mmHg}$ vs $133.33 \pm 4.58 \mathrm{mmHg}$ and MURE vs MC, $103.00 \pm 4.69 \mathrm{mmHg}$ vs $133.33 \pm 4.58$ $\mathrm{mmHg}$ on $\mathrm{GD} 18)(\mathrm{P}<0.01)$.

\section{URE alleviated the proteinuria in LPS-induced PE-like rats}

As shown in Figure 4, there was no difference in proteinuria among the five groups before GD 14. The levels of 24-h proteinuria in the PC group did not change during the research period. However, levels of 24-h proteinuria were increased in the LPS-induced PE-like rats on GD 17 and 19 compared to those in the $P C$ group $(P<0.01)$. Interestingly, these differences were ameliorated by treatment with HURE and MURE $(P<0.01)$.

\section{URE improved pregnancy outcomes in LPS-induced PE-like rats}

As shown in Table 2, the placental weight, fetal weight, and number of live pups were significantly decreased in the $M C$ group $(P<0.05)$. However, HURE treatment increased placental weight, fetal weight, and number of live pups. 


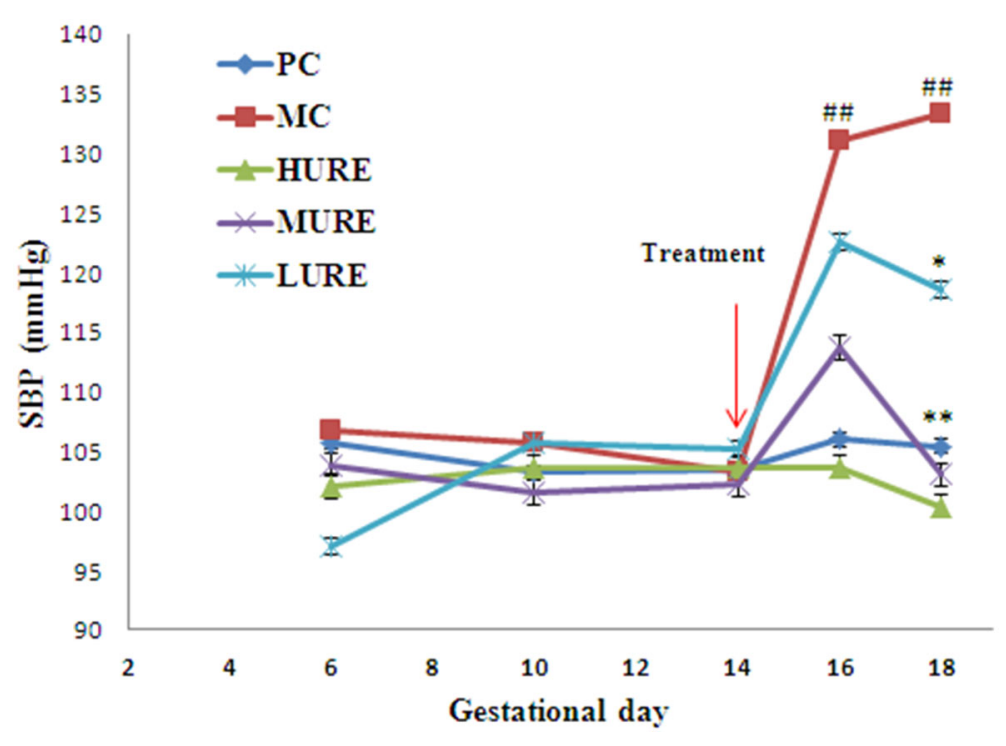

Figure 3. Uncaria rhynchophylla alkaloid extract (URE) reduced systolic blood pressures (SBP) in the lipopolysaccharide (LPS)-induced preeclampsia-like rats. SBP was measured on gestational days $6,10,14,16$, and 18 in the pregnant groups. Data are reported as means $\pm \mathrm{SE}$. ${ }^{\# \#} \mathrm{P}<0.01$ vs $\mathrm{PC}$ group, ${ }^{*} \mathrm{P}<0.05$ and ${ }^{* *} \mathrm{P}<0.01$ vs MC group (ANOVA). PC: pregnant control group; MC: LPS-treated group; HURE: LPS plus high-dose URE; MURE: LPS plus medium-dose URE; LURE: LPS plus low-dose URE.

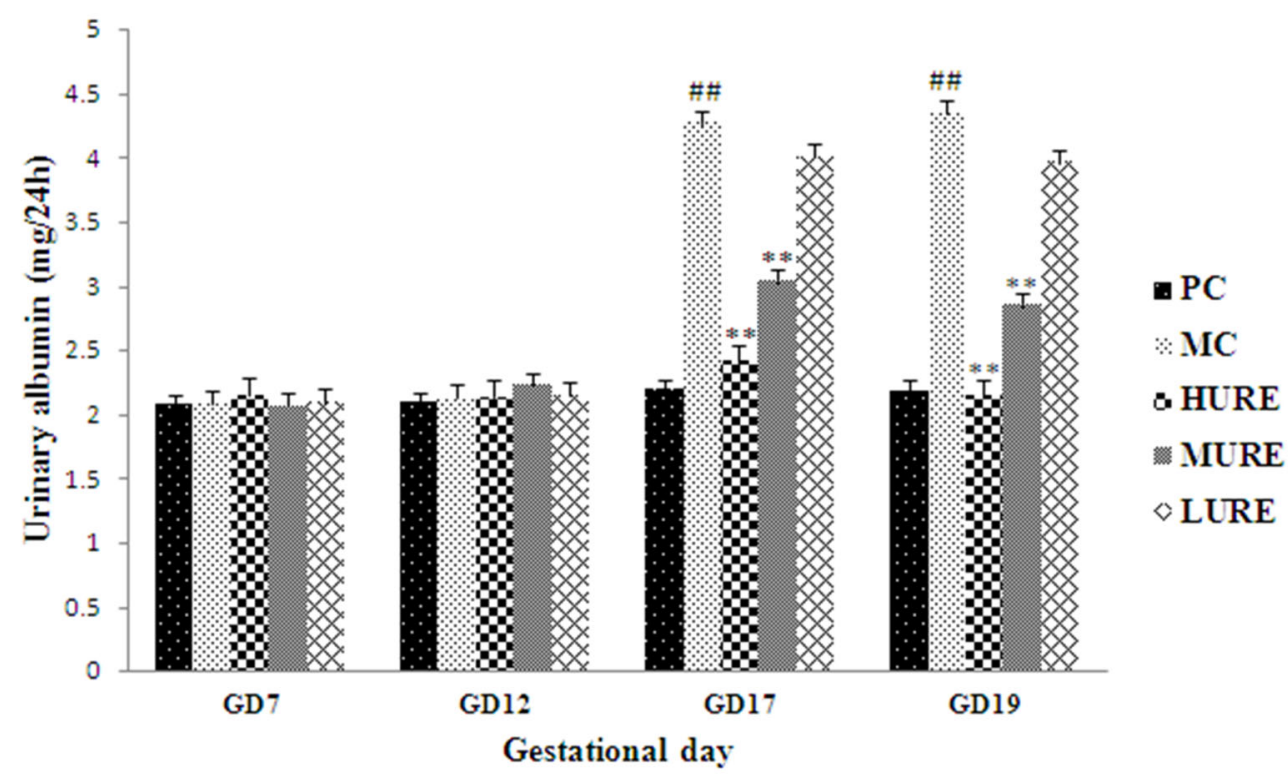

Figure 4. Uncaria rhynchophylla alkaloid extract (URE) ameliorated urinary albumin in the lipopolysaccharide (LPS)-induced preeclampsialike rats. The 24-h urinary albumin was measured on gestational day (GD) $7,12,17$, and 19. Data are reported as means \pm SE. ${ }^{\# \#} \mathrm{P}<0.01$ vs $\mathrm{PC}$ group, ${ }^{* *} \mathrm{P}<0.01$ vs MC group (ANOVA). PC: pregnant control group; MC: LPS-treated group; HURE: LPS plus highdose URE; MURE: LPS plus medium-dose URE; LURE: LPS plus low-dose URE.

These results indicated that URE can improve unfavorable pregnancy outcomes in LPS-induced PE-like rats.

\section{Effects of URE on the levels of pro-inflammatory cytokines in the serum}

Data of serum pro-inflammatory cytokines are reported in Figure 5. After infusion of LPS, the levels of pro-inflammatory cytokines (IL-6, $98.45 \pm 8.45 \mathrm{pg} / \mathrm{mL}$; IL $1-\beta, 86.65 \pm 5.93 \mathrm{pg} / \mathrm{mL}$; TNF- $\alpha, 266.93 \pm 16.46 \mathrm{pg} / \mathrm{mL}$; IFN- $\gamma, 131.64 \pm 8.45 \mathrm{pg} / \mathrm{mL}$ ) significantly increased in the $\mathrm{MC}$ group compared with the control group $(\mathrm{P}<0.01)$. The intragroup comparison showed that IL-6, IL-1 $\beta$, TNF- $\alpha$, and IFN- $\gamma$ significantly declined after treatment with HURE compared with the MC group $(P<0.01)$. 
Table 2. Pregnancy outcomes in different groups on gestational day 20.

\begin{tabular}{lccc}
\hline Group & Number of live fetuses & Mean fetal weight $(\mathrm{g})$ & Mean placental weight $(\mathrm{g})$ \\
\hline PC & $9.7 \pm 0.8$ & $4.9 \pm 0.24$ & $0.54 \pm 0.02$ \\
MC & $4.6 \pm 0.9^{\#}$ & $3.7 \pm 0.35^{\#}$ & $0.43 \pm 0.02^{\#}$ \\
HURE & $10 \pm 1.3^{*}$ & $4.4 \pm 0.33$ & $0.52 \pm 0.03$ \\
MURE & $6.2 \pm 1.0$ & $4.0 \pm 0.32$ & $0.47 \pm 0.02$ \\
LURE & $5.1 \pm 0.8$ & $4.0 \pm 0.28$ & $0.45 \pm 0.03$ \\
\hline
\end{tabular}

Data are reported as means $\pm \mathrm{SE}$ ( $\mathrm{n}=8 /$ group). ${ }^{\#} \mathrm{P}<0.05$ vs the $\mathrm{PC}$ group, ${ }^{*} \mathrm{P}<0.05$ vs the $\mathrm{MC}$ group (ANOVA). PC: pregnant control group; MC: lipopolysaccharide (LPS)-treated group; HURE: LPS plus highdose Uncaria rhynchophylla alkaloid extract (URE); MURE: LPS plus medium-dose URE; LURE: LPS plus low-dose URE.

A

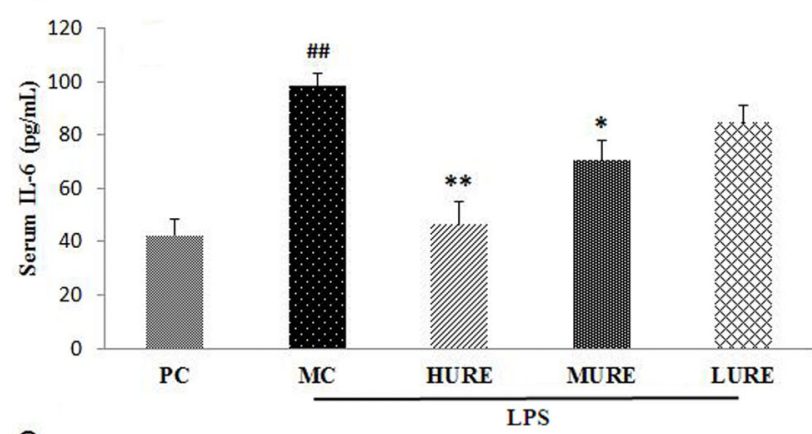

C

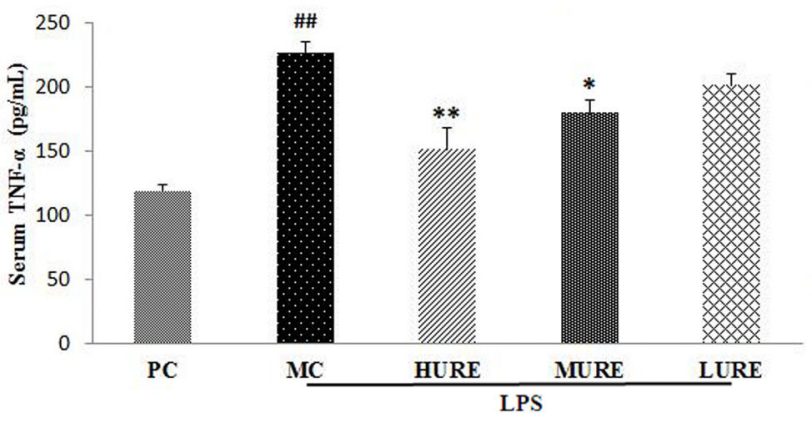

B

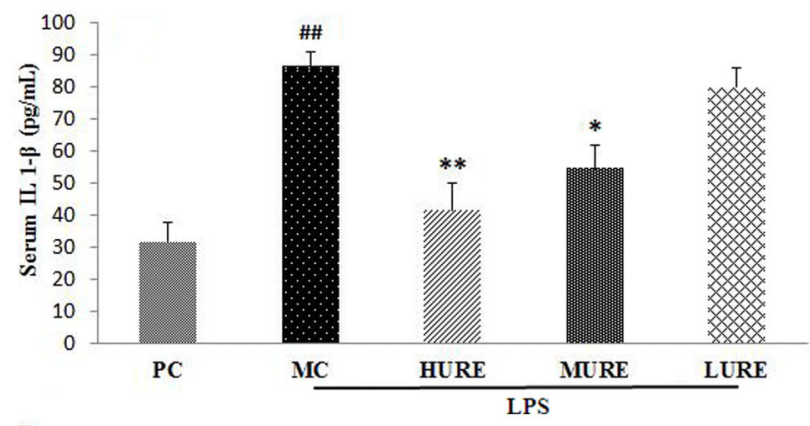

D

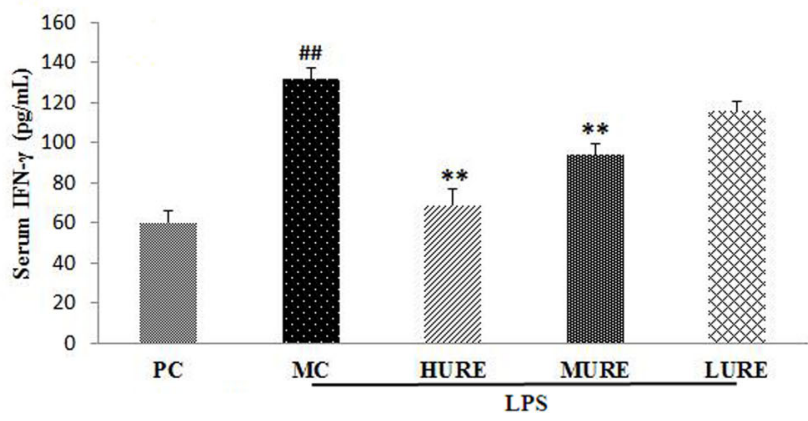

Figure 5. Serum levels of inflammatory cytokines in different groups on gestational day 20. Serum levels of interleukin (IL)-6 (A), IL $1-\beta(B)$, tumor necrosis factor (TNF)- $\alpha(\mathbf{C})$, and interferon (IFN)- $\gamma(\mathbf{D})$ were significantly higher in the MC group and lower in the Uncaria rhynchophylla alkaloid extract (URE) group. Data are reported as means $\pm \mathrm{SE}$ (n=8/group). ${ }^{\#} \mathrm{P}<0.01$ vs $\mathrm{PC}$ group, ${ }^{*} \mathrm{P}<0.05$ and ${ }^{* *} \mathrm{P}<0.01$ vs MC group (ANOVA). PC: pregnant control group; MC: LPS-treated group; HURE: LPS plus high-dose URE; MURE: LPS plus medium-dose URE; LURE: LPS plus low-dose URE.

\section{Effects of URE on the levels of pro-inflammatory cytokines in the placenta}

Data of placenta pro-inflammatory cytokines are reported in Figure 6. After injection of LPS, the levels of pro-inflammatory cytokines (IL-6, $23.15 \pm 4.37 \mathrm{pg} \cdot \mathrm{mL}^{-1}$. $\mathrm{mg}^{-1}$; IL $1-\beta, 79.15 \pm 4.32 \mathrm{pg} \cdot \mathrm{mL}^{-1} \cdot \mathrm{mg}^{-1}$; TNF- $\alpha, 11.57 \pm$ $1.19 \mathrm{pg} \cdot \mathrm{mL}^{-1} \cdot \mathrm{mg}^{-1} ; \mathrm{IFN}-\gamma, 22.09 \pm 2.78 \mathrm{pg} \cdot \mathrm{mL}^{-1} \cdot \mathrm{mg}^{-1}$ ) in the $\mathrm{MC}$ group significantly increased compared with the control group $(P<0.01)$. The intragroup comparison showed that IL-6, IL-1 $\beta$, TNF- $\alpha$, and IFN- $\gamma$ significantly declined after treatment with HURE compared with the MC group $(P<0.01)$.

\section{Pro-inflammatory cytokines mRNA expression}

As shown in Figure 7, the mRNA expressions of IL-6, IL $1-\beta, T N F-\alpha$, and IFN- $\gamma$ were up-regulated in the MC group compared to the NC group. However, HURE treatment down-regulated those expressions compared to the MC group $(P<0.01)$. Accordingly, these data indicated that pro-inflammatory cytokines mRNA expressions were 

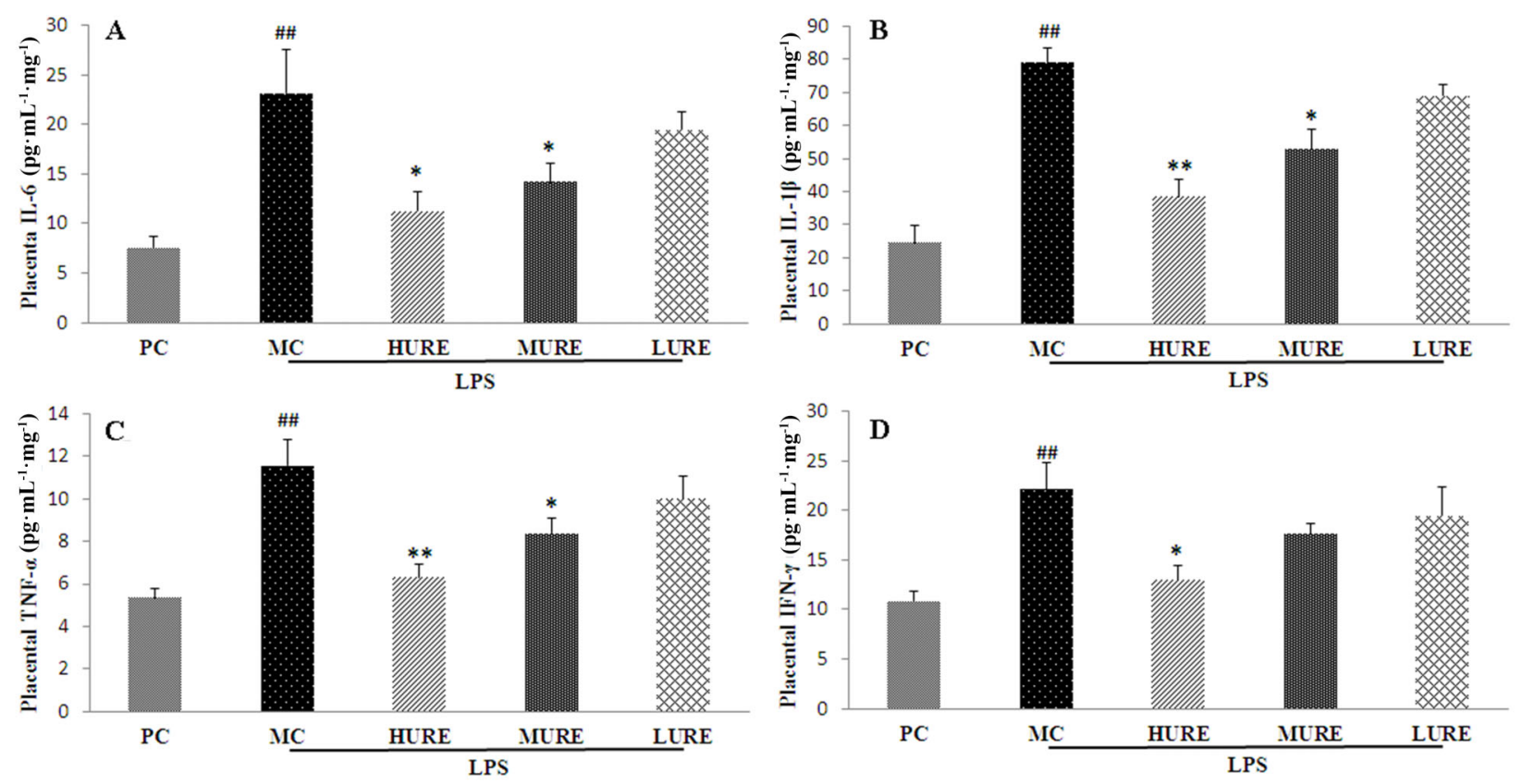

Figure 6. Placental levels of inflammatory cytokines in different groups on gestational day 20. Placental levels of interleukin IL-6 (A), IL1- $\beta$ (B), tumor necrosis factor (TNF)- $\alpha$ (C), and interferon (IFN)- $\gamma(\mathbf{D})$ were significantly higher in the MC group and lower in the Uncaria rhynchophylla alkaloid extract (URE) group. Data are reported as means $\pm S E$ ( $n=8 /$ group). ${ }^{\#} P<0.01$ vs $P C$ group, ${ }^{*} P<0.05$ and ${ }^{* *} \mathrm{P}<0.01$ vs MC group (ANOVA). PC: pregnant control group; MC: LPS-treated group; HURE: LPS plus high-dose URE; MURE: LPS plus medium-dose URE; LURE: LPS plus low-dose URE.

suppressed by HURE and thereby inflammatory response was mitigated in the LPS-treated rats.

\section{URE suppressed the LPS-induced activation of NF-кB p65 in the placenta}

As shown in Figure 8, the level of NF- $\mathrm{B}$ p 65 was increased in the LPS-induced PE rats, whereas HURE treatment decreased nuclear-positive staining compared to the MC group $(P<0.01)$.

\section{Discussion}

Indole alkaloids are the main bioactive constituents presented in $U$. rhynchophylla, and play a vital role in improving anti-inflammatory effects and reducing hypertensive effects $(12,22)$. However, there is no study linking the improvement by alkaloids from $U$. rhynchophylla to the PE-like phenotypes and pro-inflammatory cytokines caused by LPS. In this study, the purified URE improved adverse pregnancy outcomes by ameliorating PE-like symptoms and suppressing inflammation in the LPStreated rats.

$\mathrm{PE}$ is characterized by increased maternal proinflammatory cytokines, including IL- $6, \mathrm{IL}-1 \beta$, TNF- $\alpha$, and IFN- $\gamma$ (7). Previous research has exhibited that excessive inflammation plays a critical role in the pathology and pathogenesis of PE (28). There are several pregnant rat models by injection of LPS, which is a potent virulence factor in terms of pro-inflammatory property $(8,22)$. In the present study, a rat model of PE was successfully established by injecting LPS daily to pregnant rats until GD 14. The cause for the obvious elevation of blood pressure in LPS-treated rats may be due to excessive maternal proinflammatory cytokines resulting in alteration of maternal hemostasis, endothelial dysfunction, and vasoconstriction (29). In addition, LPS also increased the level of proteinuria in pregnant rats. Furthermore, the finding of declined fetal and placental weight and increased embryonic resorption in LPS-treated rats is in accordance with previous results in humans exhibiting fetal growth restriction and fetal loss in Gram-negative bacterial infections (30). These results imply that inflammation is an important mechanism in the pathogenesis of LPS-induced PE-like symptoms.

Accumulating evidence has shown that modulation of inflammation may be an effective therapy for the prevention and treatment of pregnancy complications. As vitamin D3 and aspirin possess anti-inflammatory effects, lowdose vitamin D3 and aspirin in early gestation were used for reducing the risk of PE and adverse perinatal health outcomes (31). The dried stems of $U$. rhynchophylla have been used as an antihypertensive, antipyretic, and anticonvulsant in traditional Chinese medicine. Research has also shown that $U$. rhynchophylla relieves atopic 
A

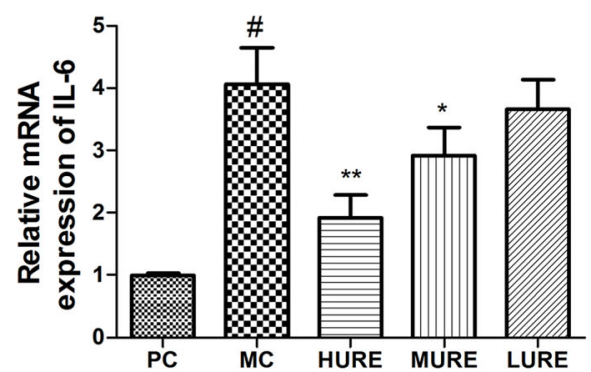

C

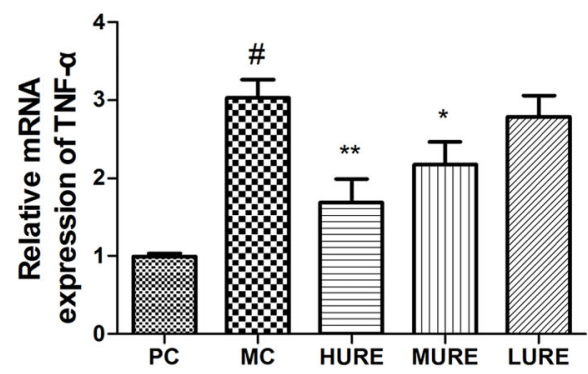

B

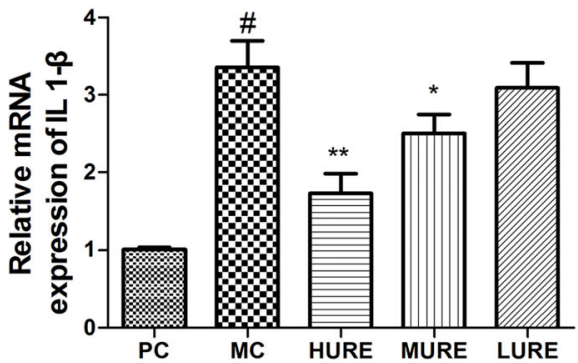

D

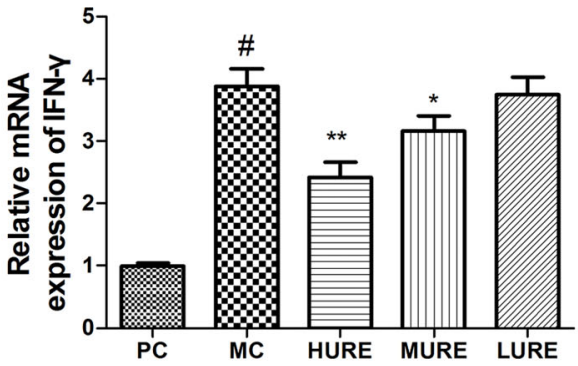

Figure 7. Relative mRNA levels of interleukin (IL)-6 (A), IL-1 $\beta$ (B), tumor necrosis factor (TNF)- $\alpha$ (C), and interferon (IFN)- $\gamma$ (D) in placenta tissue determined by qPCR. Data are reported as means $\pm \mathrm{SE}$ ( $\mathrm{n}=8 /$ group). ${ }^{\#} \mathrm{P}<0.01$ vs the $\mathrm{PC}$ group, ${ }^{*} \mathrm{P}<0.05$ and ${ }^{* *} \mathrm{P}<0.01$ vs the MC group (ANOVA). PC: pregnant control group; MC: LPS-treated group; HURE: LPS plus high-dose Uncaria rhynchophylla alkaloid extract (URE); MURE: LPS plus medium-dose URE; LURE: LPS plus low-dose URE.

A
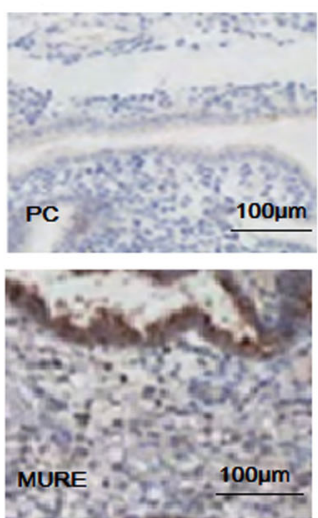
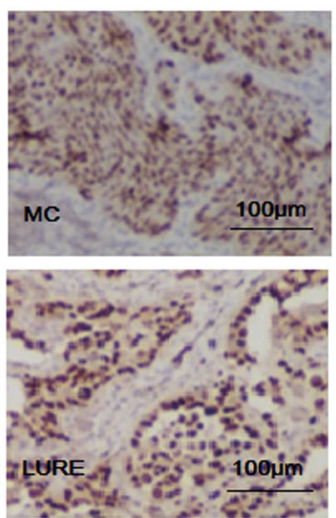
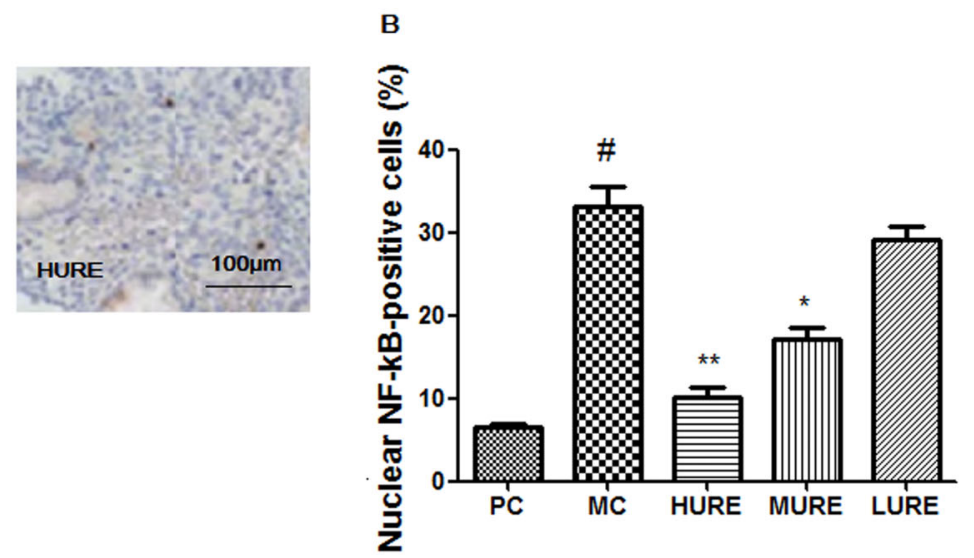

Figure 8. Uncaria rhynchophylla alkaloid extract (URE) suppressed the lipopolysaccharide (LPS)-induced activation of NF-kB p65 in the placenta tissue. Immunohistochemical staining of placental tissues (A). Original magnification: $\times 100$; scale bar $=100 \mu \mathrm{m}$. B, Percentage of nuclear NF-kB-positive cells in placenta tissue. Data are reported as means $\pm \mathrm{SE}$ ( $\mathrm{n}=8 /$ group). ${ }^{\#} \mathrm{P}<0.01$ vs $\mathrm{PC}$ group, ${ }^{*} \mathrm{P}<0.05$ and ${ }^{* *} \mathrm{P}<0.01$ vs MC group (ANOVA). PC: pregnant control group; MC: LPS-treated group; HURE: LPS plus high-dose URE; MURE: LPS plus medium-dose URE; LURE: LPS plus low-dose URE.

dermatitis (32) and the indole alkaloids of $U$. rhynchophylla have anti-inflammatory mediators such as IL-1 $\beta$, $\mathrm{NO}$, and TNF- $\alpha$. In LPS-activated N9 cells, the antiinflammatory mechanisms of alkaloids were relieved by blocking the activation of NF-KB, ERK, and p38 MAPKs (33). Due to its anti-inflammatory effect, U. rhynchophylla may be useful in the prevention and treatment of inflammation-related PE-like phenotypes. 
The present research showed that URE treatment significantly prevented the emergence of phenotypes of $P E$ in LPS-administered rats from GD14 to GD 18. Moreover, URE also improved pregnancy outcomes in the MC group, with increased fetal weight, placenta weight, and the number of live fetuses. These results suggested that URE may be an effective therapy for prevention and treatment of preeclampsia-like phenotypes and adverse pregnancy outcomes. Furthermore, in our LPS-induced rat model of PE, we found that URE effectively ameliorated urinary protein excretion and SBP, and that these changes were accompanied by suppression of the levels of pro-inflammatory cytokines in the placenta and serum. The present research supports that URE can alleviate PE and suppress inflammation to improve adverse pregnancy outcomes in rats.

The phytochemical analysis of the URE revealed the presence of indole alkaloids, including isorhynchophylline, yohimbine, $3 \alpha$-dihydrocadambine, raubasine, hirsuteine, and hirsutine. Previous research has disclosed that indole alkaloids extracts are responsible for anti-inflammatory

\section{References}

1. Roberts JM, Redman CWG. Pre-eclampsia: more than pregnancy-induced hypertension. The Lancet 1993; 341: 1447-1451, doi: 10.1016/0140-6736(93)90889-O.

2. Knight M, UKOSS. Eclampsia in the United Kingdom 2005. BJOG 2007; 114: 1072-1078, doi: 10.1111/j.1471-0528. 2007.01423.x.

3. Ghulmiyyah L, Sibai B. Maternal mortality from preeclampsia/ eclampsia. Semin Perinatol 2012; 36: 56-59, doi: 10.1053/ j.semperi.2011.09.011.

4. Rodie VA, Freeman DJ, Sattar N, Greer IA. Pre-eclampsia and cardiovascular disease: metabolic syndrome of pregnancy? Atherosclerosis 2004; 175: 189-202, doi: 10.1016/ j.atherosclerosis.2004.01.038.

5. Laresgoiti-Servitje E. A leading role for the immune system in the pathophysiology of preeclampsia. J Leukoc Biol 2013; 94: 247-257, doi: 10.1189/jlb.1112603.

6. Sacks G, Sargent I, Redman C. An innate view of human pregnancy. Immunology Today 1999; 20: 114-118, doi: 10.1016/S0167-5699(98)01393-0.

7. Pinheiro MB, Martins-Filho OA, Mota AP, Alpoim PN, Godoi LC, Silveira AC, et al. Severe preeclampsia goes along with a cytokine network disturbance towards a systemic inflammatory state. Cytokine 2013; 62: 165-173, doi: 10.1016/ j.cyto.2013.02.027.

8. Gong $\mathrm{P}$, Liu M, Hong G, Li Y, Xue P, Zheng M, et al. Curcumin improves LPS-induced preeclampsia-like phenotype in rat by inhibiting the TLR4 signaling pathway. Placenta 2016; 41: 45-52, doi: 10.1016/j.placenta.2016.03.002.

9. Soares de Moura R, Resende ÂC, Moura AS, Maradei MF. Protective action of a hydroalcoholic extract of a vinifera grape skin on experimental preeclampsia in rats. Hypertens Pregnancy 2007; 26: 89-100, doi: 10.1080/1064195060 1147960.

10. Ndagijimana A, Wang X, Pan G, Zhang F, Feng H, Olaleye $O$. A review on indole alkaloids isolated from Uncaria and anti-hypertensive activities, with which our experimental results are in accordance. Mitraphylline and isomitraphylline from Uncaria tomentosa extracts inhibited the production of pro-inflammatory cytokines in vitro (34). Rhynchophylline and isorhynchophylline from $U$. rhynchophylla extracts suppressed inflammatory mediators by blocking iNOS protein expression and blocking the activation of $\mathrm{NF}-\kappa \mathrm{B}, \mathrm{ERK}$, and p38 MAPKs in N9 cells (33). $3 \alpha$-dihydrocadambine showed dose-dependent anti-hypertensive and hypotensive activities in conscious spontaneous rats and in anesthetized normotensive rats (35).

In conclusion, our research showed that URE improved $P E$ signs and inhibited pro-inflammatory cytokines in a PE rat model. URE is a native medicine with anti-inflammatory and anti-hypertensive properties, which could be attributed to indole alkaloids. These findings revealed a novel therapeutic use of URE for preventing excessive proinflammatory cytokines in pregnancy complications. However, the underlying mechanisms need to be elucidated in further research.

rhynchophylla and their pharmacological studies. Fitoterapia 2013; 86: 35-47, doi: 10.1016/j.fitote.2013.01.018.

11. Ho TY, Tang NY, Hsiang CY, Hsieh CL. Uncaria rhynchophylla and rhynchophylline improved kainic acid-induced epileptic seizures via IL-1 $\beta$ and brain-derived neurotrophic factor. Phytomedicine 2014; 21: 893-900, doi: 10.1016/ j.phymed.2014.01.011.

12. Li $\mathrm{Y}$, Jiang $\mathrm{YH}$, Yang $\mathrm{CH}$, Sun JC, Wang MM, Yang WQ. Enhanced protective effect of the combination of uncaria and semen raphani on vascular endothelium in spontaneously hypertensive rats. Evid Based Complement Alternat Med 2015; 2015: 358352, doi: 10.1155/2015/358352.

13. Hsieh CL, Chen MF, Li TC, Li SC, Tang NY, Hsieh CT, et al. Anticonvulsant effect of Uncaria rhynchophylla (Miq) Jack. in rats with kainic acid-induced epileptic seizure. Am J Chin Med 1999; 27: 257-264, doi: 10.1142/S0192415X9900 029X.

14. Suk K, Kim SY, Leem K, Kim YO, Park SY, et al. Neuroprotection by methanol extract of Uncaria rhynchophylla against global cerebral ischemia in rats. Life Sci 2002; 70: 2467-2480, doi: 10.1016/S0024-3205(02)01534-5.

15. Kang TH, Murakami $\mathrm{Y}$, Matsumoto K, Takayama H, Kitajima $\mathrm{M}$, Aimi N, et al. Rhynchophylline and isorhynchophylline inhibit NMDA receptors expressed in Xenopus oocytes. Eur J Pharmacol 2002; 455: 27-34, doi: 10.1016/S0014-2999(02) 02581-5.

16. Kim JH, Bae $\mathrm{CH}$, Park SY, Lee SJ, Kim Y. Uncaria rhynchophylla inhibits the production of nitric oxide and interleukin- $1 \beta$ through blocking nuclear factor $\kappa \mathrm{B}$, Akt, and mitogen-activated protein kinase activation in macrophages. J Med Food 2010; 13: 1133-1140, doi: 10.1089/jmf. 2010.1128.

17. Sharma A, Satyam A, Sharma JB. Leptin, IL-10 and inflammatory markers (TNF- $\alpha, \mathrm{IL}-6$ and IL- 8 ) in pre-eclamptic, normotensive pregnant and healthy non-pregnant women. 
Am J Reprod Immunol 2007; 58: 21-30, doi: 10.1111/j.16000897.2007.00486.x.

18. Peraçoli JC, Rudge MV, Peraçoli MT. Tumor necrosis factor-alpha in gestation and puerperium of women with gestational hypertension and pre-eclampsia. Am J Reprod Immunol 2007; 57: 177-185, doi: 10.1111/j.1600-0897.2006. 00455.x.

19. Kunnen A, van Pampus MG, Aarnoudse JG, van der Schans CP, Abbas F, Faas MM. The effect of Porphyromonas gingivalis lipopolysaccharide on pregnancy in the rat. Oral Dis 2014; 20: 591-601, doi: 10.1111/odi.12177.

20. Cotechini T, Komisarenko M, Sperou A, Macdonald-Goodfellow $\mathrm{S}$, Adams M, Graham $\mathrm{CH}$. Inflammation in rat pregnancy inhibits spiral artery remodeling leading to fetal growth restriction and features of preeclampsia. J Exp Med 2014; 211: 165-179, doi: 10.1084/jem.20130295.

21. Collins S, Ramsay M, Slack MP, Campbell H, Flynn S, Litt D, et al. Risk of invasive Haemophilus influenzae infection during pregnancy and association with adverse fetal outcomes. JAMA 2014; 311: 1125-1132, doi: 10.1001/jama. 2014.1878.

22. Xue $P$, Zheng M, Gong P, Lin C, Zhou J, Li Y, et al. Single administration of ultra-low-dose lipopolysaccharide in rat early pregnancy induces TLR4 activation in the placenta contributing to preeclampsia. PLoS ONE 2015; 10: e0124001, doi: 10.1371/journal.pone.0124001.

23. Barrera D, Díaz L, Noyola-Martínez N, Halhali A. Vitamin D and inflammatory cytokines in healthy and preeclamptic pregnancies. Nutrients 2015; 7: 6465-6490, doi: 10.3390/ nu7085293.

24. Liu J, Li Q, Liu R, Yin $Y$, Chen $X$, Bi K. Enrichment and purification of six Aconitum alkaloids from Aconiti kusnezoffii radix by macroporous resins and quantification by HPLC-MS. J Chromatogr B Analyt Technol Biomed Life Sci 2014; 960: 174-181, doi: 10.1016/j.jchromb.2014. 04.034.

25. Liu Y, Yang J, Bao J, Li X, Ye A, Zhang G, et al. Activation of the cholinergic anti-inflammatory pathway by nicotine ameliorates lipopolysaccharide-induced preeclampsia-like symptoms in pregnant rats. Placenta 2017; 49: 23-32, doi: 10.1016/j.placenta.2016.11.003.

26. Zhang Y, Hua Z, Zhang K, Meng K, Hu Y. Therapeutic Effects of Anticoagulant Agents on Preeclampsia in a Murine Model Induced by Phosphatidylserine/Phosphatidylcholine Microvesicles. Placenta 2009; 30: 1065-1070, doi: 10.1016/ j.placenta.2009.09.004.
27. Zhao B, Huang Y, Chen Q, Chen Q, Miao H, Zhu S, et al. Characteristic component profiling and identification of different Uncaria species based on high-performance liquid chromatography-photodiode array detection tandem ion trap and time of flight mass spectrometry coupled with rDNA ITS sequence. Biomed Chromatogr 2018; 32: e4119, doi: 10.1002/bmc.4119.

28. Hartley JD, Ferguson BJ, Moffett A. The role of shed placental DNA in the systemic inflammatory syndrome of preeclampsia. Am J Obstet Gynecol 2015; 213: 268-277, doi: 10.1016/j.ajog.2015.03.026.

29. Shaw J, Tang Z, Schneider H, Saljé K, Hansson SR, Guller $S$. Inflammatory processes are specifically enhanced in endothelial cells by placental-derived TNF- $\alpha$ : Implications in preeclampsia (PE). Placenta 2016; 43: 1-8, doi: 10.1016/ j.placenta.2016.04.015.

30. Collins S, Ramsay M, Slack MP, Campbell H, Flynn S, Litt D, et al. Risk of invasive Haemophilus influenzae infection during pregnancy and association with adverse fetal outcomes. JAMA 2014; 311: 1125-1132, doi: 10.1001/jama.2014.1878.

31. Henderson JT, Whitlock EP, O'Connor E, Senger CA, Thompson JH, Rowland MG. Low-dose aspirin for prevention of morbidity and mortality from preeclampsia: a systematic evidence review for the U.S. Preventive Services Task Force. Ann Intern Med 2014; 160: 695-703, doi: 10.7326/ M13-2844.

32. Kim DY, Jung JA, Kim TH, Seo SW, Jung SK, Park CS. Oral administration of Uncariae rhynchophylla inhibits the development of DNFB-induced atopic dermatitis-like skin lesions via IFN- $\gamma$ down-regulation in NC/Nga mice. J Ethnopharmacol 2009; 122: 567-572, doi: 10.1016/j.jep.2008.12.029.

33. Yuan D, Ma B, Yang JY, Xie YY, Wang L, Zhang LJ, et al. Anti-inflammatory effects of rhynchophylline and isorhynchophylline in mouse N9 microglial cells and the molecular mechanism. Int Immunopharmacol 2009; 9: 1549-1554, doi: 10.1016/j.intimp.2009.09.010.

34. Azevedo BC, Morel LJF, Carmona F, Cunha TM, Contini SHT, Delprete PG, et al. Aqueous extracts from Uncaria tomentosa (Willd. ex Schult.) DC. reduce bronchial hyperresponsiveness and inflammation in a murine model of asthma. J Ethnopharmacol 2018; 218: 76-89, doi: 10.1016/j.jep.2018. 02.013.

35. Aisaka K, Hattori Y, Kihara T, Ishihara T, Endo K, Hikino H. Hypotensive action of 3alpha-dihydrocadambine, an indole alkaloid glycoside of uncaria hooks1. Planta Med 1985; 51: 424-427, doi: 10.1055/s-2007-969538. 